\title{
Population Analysis of Seyfert Galaxies in the Coma-Abell 1367 Supercluster
}

\author{
Megan Jones, Eric Wilcots \\ University of Wisconsin-Madison ${ }^{1}$
}

\begin{abstract}
We are studying the population of active galaxies residing both in and out of groups along the Coma-Abell 1367 supercluster to look at the occurrence of Seyfert galaxies. We are also measuring the level of activity, as defined by active galactic nuclei (AGN) and star formation rates in the galaxy groups. Our goal is then to relate this information to determine any environmental correlation of these two features. We report on the distribution of Seyfert galaxies as a function of environment across the supercluster and probe the characteristics of the population of groups that currently host at least one Seyfert.
\end{abstract}

\section{Introduction}

AGN feedback. One of the major unresolved issues in our understanding the growth of galaxies, their central black holes, and their surrounding structures is the role of feedback. Feedback is the term for energy deposited back into the surroundings from the formation of stars or central black holes. Feedback is also a potential source for additional heating of intergalactic gas. It is now well established that the intergalactic gas in galaxy groups is significantly hotter than can be explained by the pure gravitational infall of baryons into the potential well (Borgani et al 2002, Jeltema et al 2006). The source of this excess heat is believed to be either starburst driven galactic outflows or outflows from active galactic nuclei (Lloyd-Davies, Ponman, \& Cannon 2002, Nath \& Roychowdhurry 2002). This unexplained heating of the intergalactic gas between groups is a major puzzle for astronomers. In the hopes of finding a possible source of intergalactic heating, I am conducting a population analysis of the Seyfert galaxies throughout a section of the Coma-Abell 1367 supercluster. A Seyfert galaxy is a type of active galactic nucleus (AGN), the name given to a galaxy that hosts an actively accreting supermassive black hole in its nucleus/center. Seyfert galaxies tend to be spirals, and are more widely distributed than elliptical galaxies. Our own Milky Way was probably once a Seyfert. In an effort to analyze intergalactic heating, I am investigating the properties of these AGN phenomena.

What is a Seyfert? Seyfert galaxies, named for astronomer Carl K. Seyfert, are a type of AGN characterized by strong, broad emission lines (Seyfert 1943), with an actively accreting black hole within the nucleus (Weedman 1977). These broad emission lines, in the case of Seyferts, is defined by Weedman as $10^{3} \mathrm{~km} \mathrm{sec}^{-1}$ (Weedman 1977). Seyfert galaxies are identified as objects with high values (greater than 2-3) of the ratio of OIII to $\mathrm{H}_{\beta}$ (Kauffmann et al. 2003).

\footnotetext{
${ }^{1}$ The Wisconsin Space Grant Consortium funded this research, and we are very appreciative of their contribution.
} 
$R, A=182.32468, D E C=24.96755, M J D=54484$, Plate=2656, Fibs $=151$

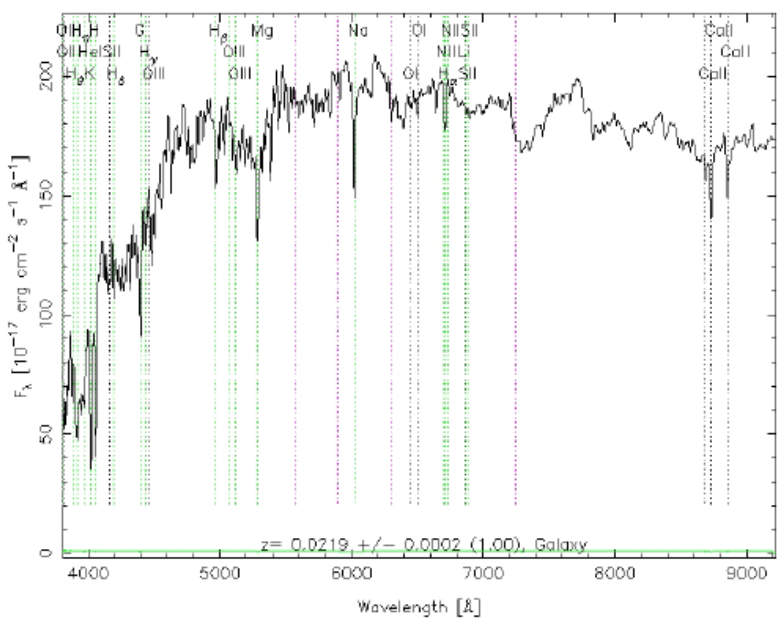

$\mathrm{RA}=178.52039, \mathrm{DEC}=27.87424, \mathrm{MJD}=53793, \mathrm{Flate}=2223$, Fiber $=629$

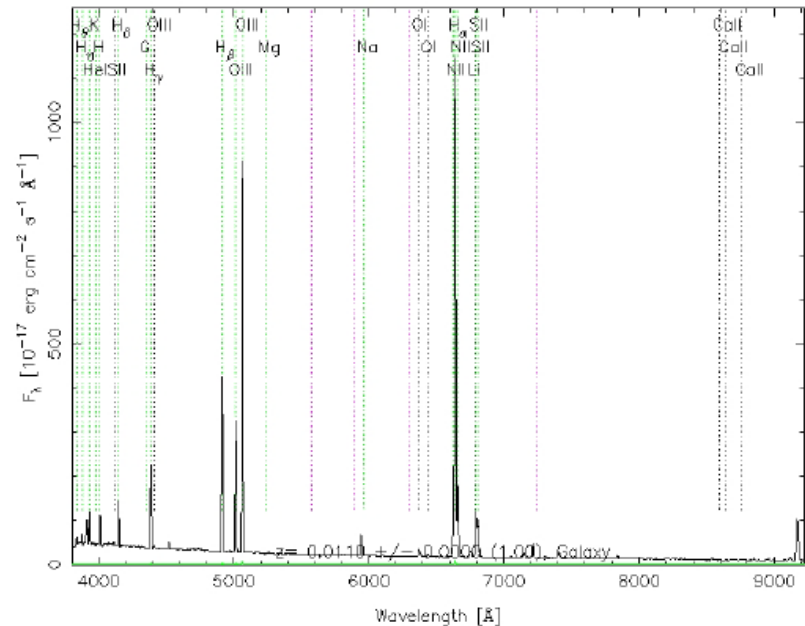

Figure 1. Galactic spectra obtained from SDSS. The spectrum on the left belongs to a non-AGN from 2MASS group 698, the one on the right is from a Seyfert galaxy in 2MASS group 683. The Seyfert spectrum clearly differs in appearance, with the broad emission lines and the 2:1 ratio of the OIII line to the $H_{\beta}$ line. The image below belongs to a starburst galaxy in the SDSS group 17426. It is very similar in appearance to the Seyfert spectrum, the main indicator being the stronger $H_{\beta}$ line.

$\mathrm{RA}=195.53284, \mathrm{DEC}=27.64831, \mathrm{MJD}=54153$, Plate $=2242$, Fiber $=437$

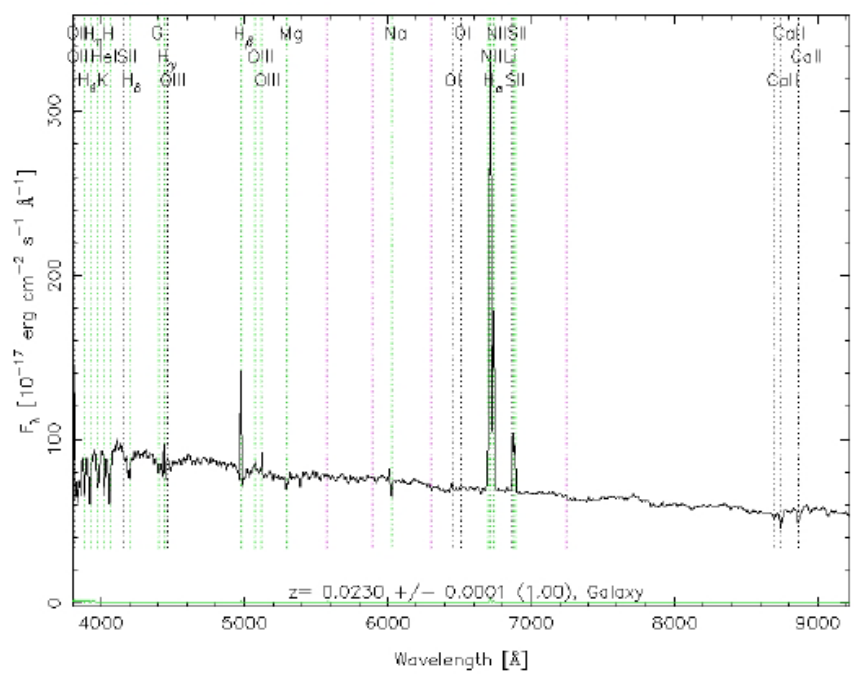

Figure 2. Spectrum of a starburst galaxy in SDSS group 17426.

\section{Welcome to the Supercluster!}

Meet Coma-Abell 1367. The supercluster spans a right ascension range from $11 \mathrm{~h}$ to $14 \mathrm{~h}$ and a range in declination from $24 \mathrm{~d}$ to $30 \mathrm{~d}$, connecting the large clusters Coma and Abell 1367. The supercluster contains regions of denser groups as well as a less dense field near the center. We have compiled a catalog of the galaxy groups and group members in the supercluster, with the data sampling regions from the densest parts of Coma to the "field" in the middle of the supercluster. Our group list comes from the 2MASS galaxy catalog as assembled by Crook et al. 2007. With the groups identified, we used the Sloan Digital Sky Survey (SDSS) to identify individual members of each group, as well as galaxies identified from the SDSS redshift survey 
(Berlind et al. 2006), producing a catalog with over 1000 spectroscopically identified galaxies. The SDSS galaxies come from the Mr18 sample, which is accessible online (http://lss.phy.vanderbilt.edu/groups/dr7/).

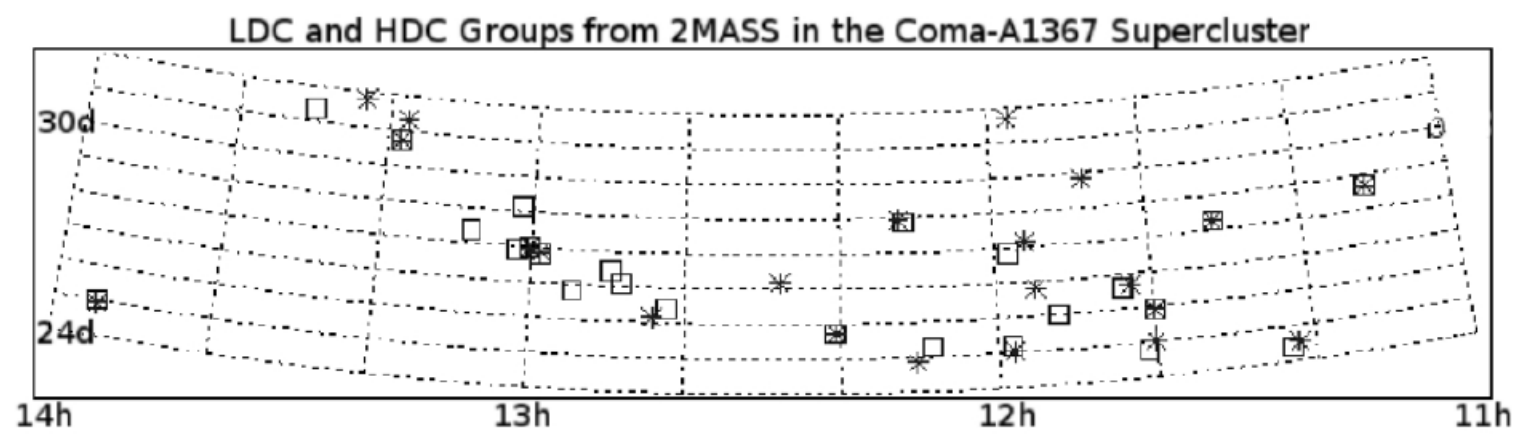

Figure 3. The positions of the 2MASS galaxy groups on the sky.

\section{AGN Activity within galaxy groups.}

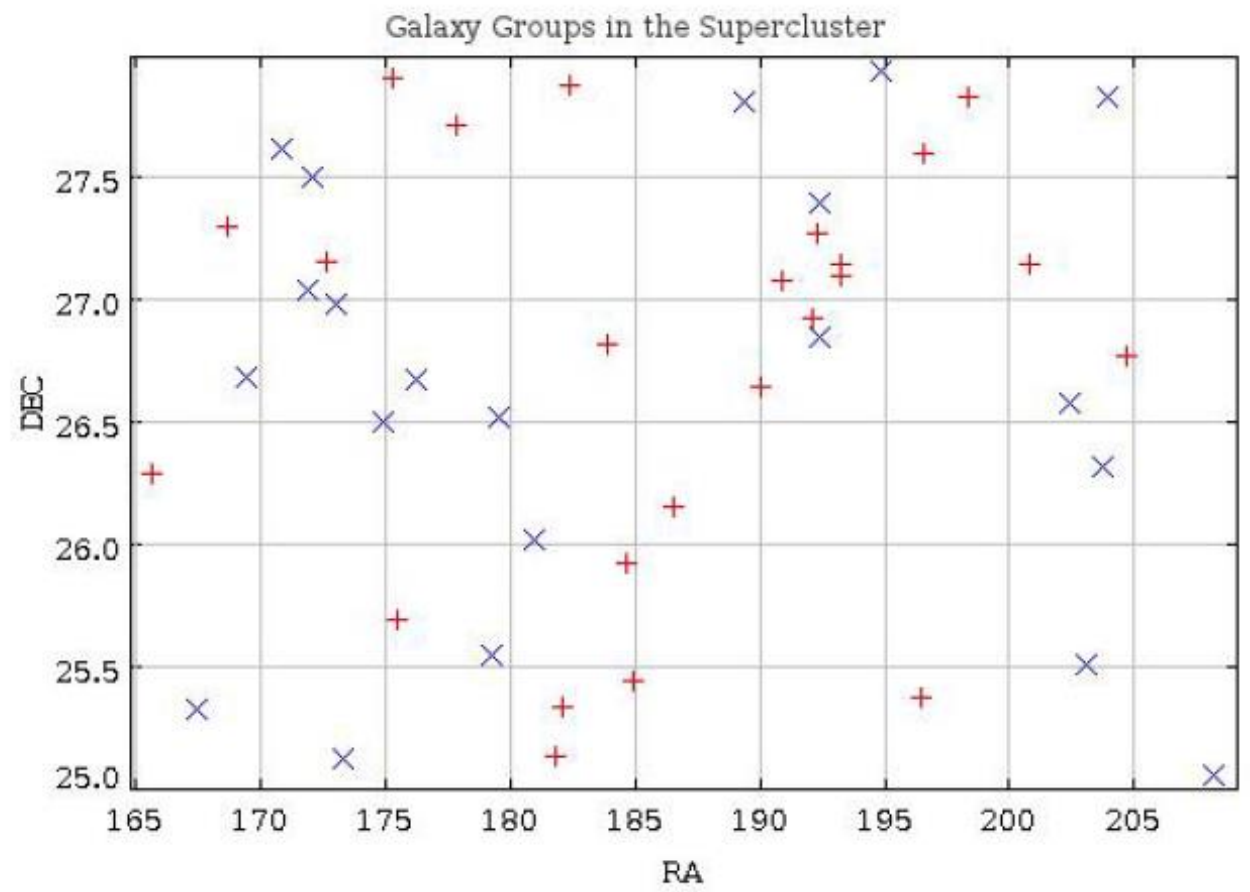

Figure 4. The galaxy groups in the Coma-Abell 1367 supercluster. The crosses represent groups with no activity, the X's represent groups hosting at least one Seyfert.

Having acquired the list of galaxies from 2MASS and the SDSS database, I marked the position of each group and noted which groups had no Seyferts, one Seyfert, and multiple Seyferts. With the identified groups, the number of catalogued Seyferts comes to about 40 confirmed Seyferts. I have plotted the individual groups and identified the location of the Seyferts within the groups to discover any trend. 

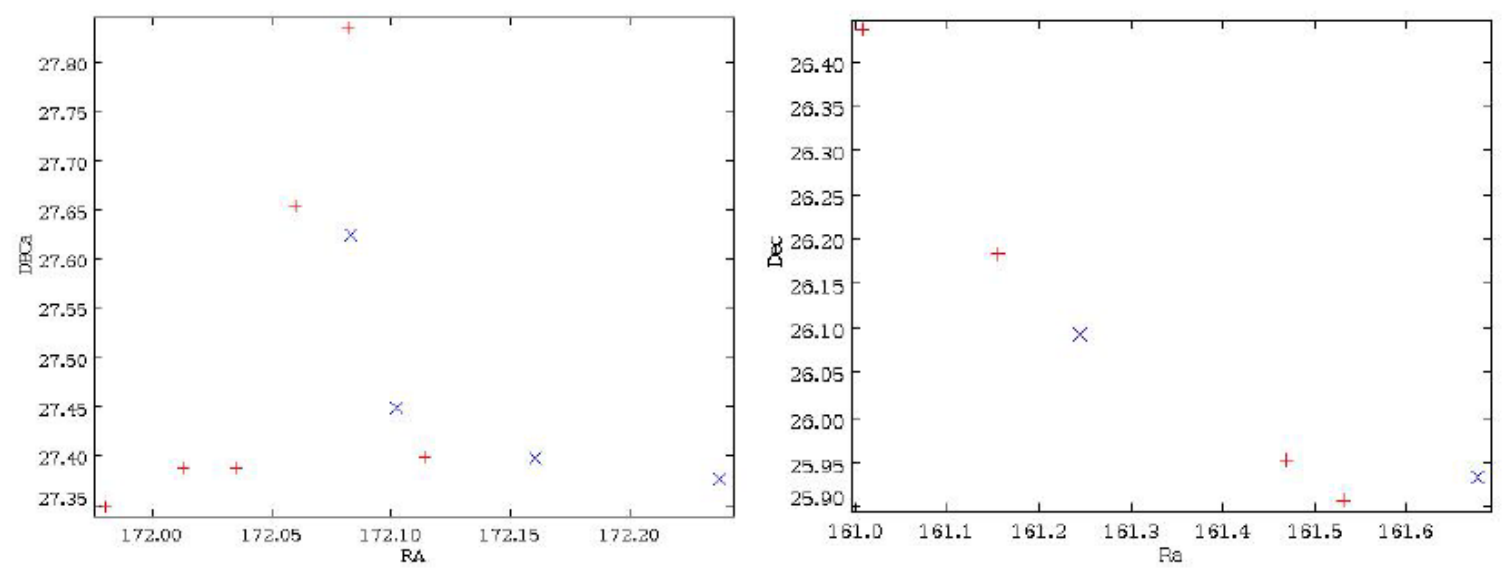

Figure 5. The positions of Seyferts in SDSS groups 19296 (left) and 19286 (right). The x's indicate Seyfert galaxies, crosses non-Seyferts. Group 17426, the heart of the Coma cluster, is shown below.

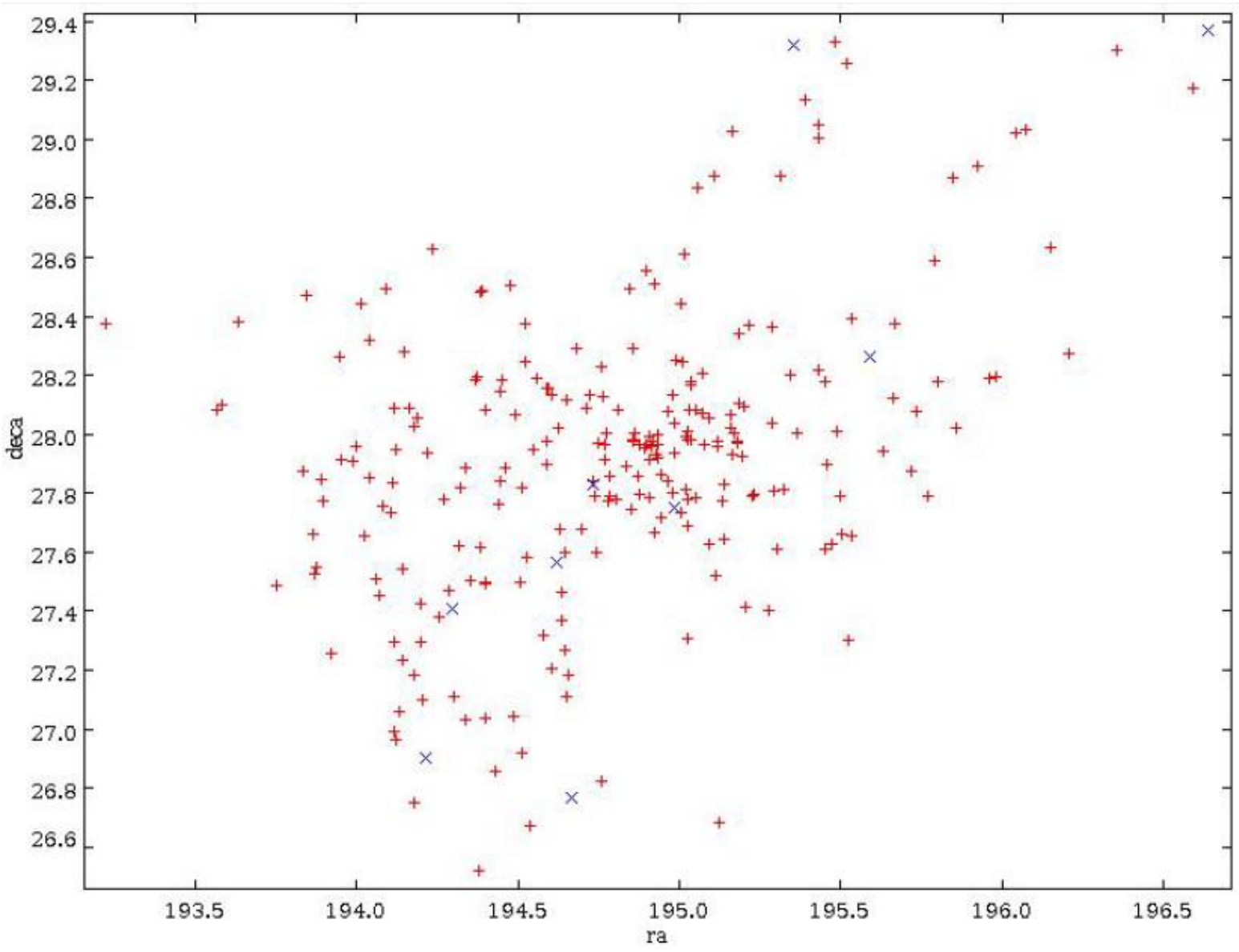

Figure 6. SDSS group 17426, the Coma cluster. 
The volume. With a somewhat clearer picture of the groups in this section of the Coma cluster, I started looking at the larger environment in which groups find themselves. I want to know what environmental factors contribute to AGN activity in galaxies, and why some groups are more likely to host multiple Seyferts while some groups have none. I used the SDSS database compile a list of Seyfert galaxies that are not positioned in groups in order to get a more unbiased sample of Seyferts. With this wider range of environmental conditions involving Seyfert galaxies, I have as unbiased of a data range as I can hope to obtain. Using the defining features of a Seyfert mentioned above, I used the line strengths of OIII and $\mathrm{H}_{\beta}$ to identify AGN in the volume that may not be situated in groups. The frequency of occurrence of Seyferts is well matched to that of the group environment, showing about a $9 \%$ contribution to the population.

Using the SDSS tool CasJobs, I retrieved the strengths for the NII, OIII, $\mathrm{H}_{\alpha}$, and $\mathrm{H}_{\beta}$ emission lines of all of the galaxies in the volume. Following the procedure by Moric et al. 2010 (Kauffmann et al. 2003; Kewley et al. 2001, 2006), I created optical spectroscopic diagnostic diagrams that separate emission-line galaxies into star-forming galaxies and AGNs. The equation for this distinction can be seen below (Kauffman et al. 2003).

$$
\log \left([O I I I] / H_{\beta}\right)>0.61 /\left[\log \left(N I I / H_{\alpha}\right)-0.05\right]+1.3(1)
$$

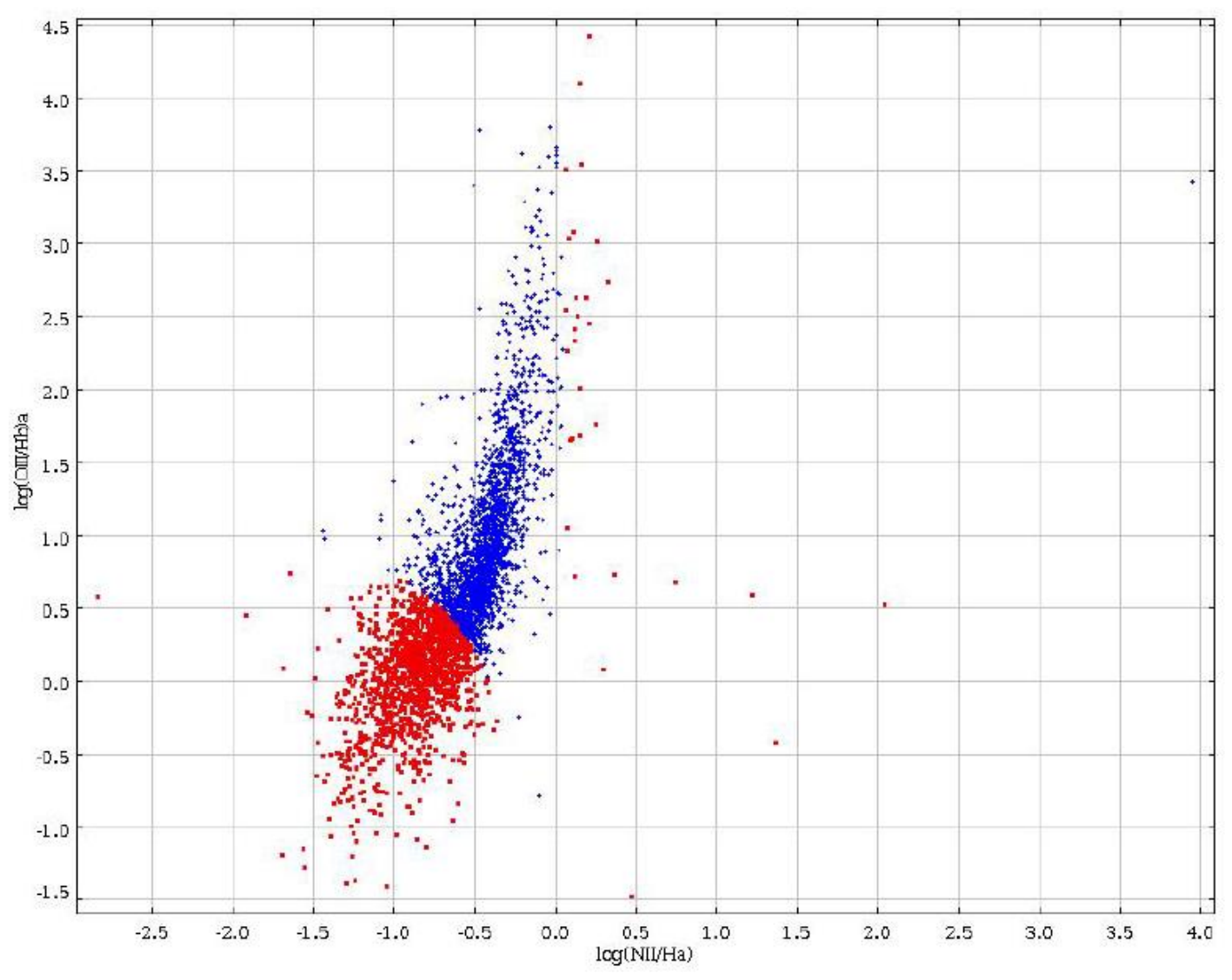

Figure 7. Optical spectroscopic diagnostic diagrams (Moric et al. 2010, Kauffmann et al. 2003, Kewley et al. 2001). Following the demarcation by Kauffmann, the dots correspond to starburst galaxies, the crosses to Seyfert galaxies. The non-Seyfert galaxies with higher ratios of NII to $H_{\alpha}$ toward the right side of the plot are most likely LINERs. 


\section{Conclusion}

Of the 66 groups sampled, there are 11groups containing one Seyfert and 6 groups containing multiple Seyferts, which leaves 49 groups without any activity. The 2MASS group 723 contains the most Seyferts by fraction: $37 \%$ of its galaxy members are classified as Seyfert galaxies. We have yet to determine whether the occurrence of Seyferts is dependent on environmental factors. So far, we have not identified a correlation between environment and AGN activity. AGN activity occurs pretty evenly both within and outside of galaxy groups. However, there does seem to be a bit of a correlation between the size of the group and the percentage of Seyfert activity; SDSS group 17426 with over 250 members has a much smaller amount of Seyferts by percentage (9 Seyferts) than the other groups with 10 or less members. We will be investigating this correlation in continuing research.

\section{References}

Abazajian, K. N., et al. 2009, ApJS, 182, 543

Berlind et al. 2006 ApJ 16238 A

Borgani, S., Governato, F., Wadsley, J., Menci, N., Tozzi, P., Quinn, T., Stadel, J., \& Lake, G. 2002, MNRAS, 336, 409

Crook, A.C., Huchra, J.P., Martimbeau, N., Masters, K.L., Jarrett, T., \& Macri, L.M. 2007, ApJ, 655, 790

Freeland, E., Stilp, A., \& Wilcots, E. 2009, AJ, 138, 295

Jeltema, T.E., Mulchaey, J.S., Lubin, L.M., Rosati, P., \& Bohringer, H. 2006, ApJ, 649, 649

Kauffmann, G. et al. Mon. Not. R. Astron. Soc. 346, 1055-1077 (2003).

Lloyd-Davies, E.J., Ponman, T.J., Cannon, D.B., 200, MNRAS, 315, 689

Moric, I. et al. ApJ 724: 779-790. 2010.

Nath, B.B., \& Roychowdhury, S. 2002, MNRAS, 333, 145

Osterbrock, D.E.: Itextit\{Astrophysics of Gaseous Nebulae and Active Galactic Nuclei\}, 1989

Schmitt, H.R., Ulvestad, J.S., Antonucci, R.J., \& Kinney, A.L. 2001, ApJ, 132, 199

Weedman, D. 1977, A\&A, 15, 69 